Emir. J. Food Agric. 2014. 26 (10): 844-852

doi: 10.9755/ejfa.v26i10.18503

http://www.ejfa.info/

Food Science and Nutrition

REGULAR ARTICLE

\title{
Genotypic, grain morphological and locality variation in rice phytate content and phytase activity
}

\author{
H. H. Lee ${ }^{1}$, C. F. J. Bong ${ }^{1}$, S. P. Loh ${ }^{2,3}$, S. R. Sarbini ${ }^{1}$ and P. H. Yiu ${ }^{1 *}$ \\ ${ }^{1}$ Faculty of Agriculture and Food Sciences, Universiti Putra Malaysia, 97000 Bintulu, Sarawak, Malaysia \\ ${ }^{2}$ Faculty of Medicine and Health Sciences, Universiti Putra Malaysia, 43400 UPM Serdang, Selangor, Malaysia \\ ${ }^{3}$ Laboratory of Molecular Biomedicine, Institute of Bioscience, Universiti Putra Malaysia, 43400 UPM Serdang, \\ Selangor, Malaysia
}

\begin{abstract}
Phytate complexes in whole grain rice are indigestible by human but can be broken down by endogenous phytase enzyme. The inherent phytate content and phytase activity could influence the nutritional quality of whole grain rice. This work aims to determine and identify their variability with genotypes, growing areas and grain morphology. It was found that the whole rice grain was largely high in phytate content (18.20 to 32.36 $\mathrm{g} / \mathrm{kg}$ ) but low in phytase activity (4.77 to $102.65 \mathrm{U} / \mathrm{kg}$ ), with significant variation among cultivars. Phytate content was marginally different between growing locations but, with no significant difference among their genotypes and grain morphology. This variation could be due to locality factors such as cropping and fertilization practices in the cultivation site. Meanwhile, phytase activity appeared to be determined by genotype, grain width and grain length-to-width ratio. A relatively high phytase activity could be selected from the whole grain rice based on rounded grain and in the genotypic category of L. These types of rice cultivars could reduce the inherent phytate level and improve the nutritional quality of the whole grain rice.
\end{abstract}

Key words: Genotype, Grain morphology, Locality, Phytase activity, Phytate, Whole grain rice

\section{Introduction}

Whole grain diet is well recognized for counteracting the diet-related diseases and promoting good health (Shahidi, 2009). Whole rice grain supplies not only calories but contains more vitamins, minerals and fiber than its processed equivalents (Champagne et al., 2004; Jones and Engleson, 2010). A wide range of phytochemicals in the rice bran serves as good source of biomedical components for consumers. Nevertheless, they are often either beneficial or deleterious, depending on the dosage and their chemical compositions.

Phytic acid (myo-inositol-1,2,3,4,5,6-hexakisdihydrogen-phosphate, IP6), is a strong chelating compound, naturally deposited in plant tissues as phytates in globoids (Raboy et al., 2000; Kumar et

Received 02 July 2014; Revised 02 August 2014; Accepted 12 August 2014; Published Online 16 September 2014

*Corresponding Author

P. H. Yiu

Faculty of Agriculture and Food Sciences, Universiti Putra Malaysia, 97000 Bintulu, Sarawak, Malaysia

Email: yiuph@upm.edu.my al., 2010). The interactions of phytic acid with food components reduce the minerals including phosphorus, protein and starch availability for human body absorption (Oatway et al., 2001; Raboy et al., 2001). However, it provides therapeutic effects under gastro-intestinal conditions by reducing the iron and zinc mediated oxidative stress and blood sugar response, resulting in antioxidant, anti-carcinogenic and anti-diabetic properties (Graf and Empson, 1987; Norazalina et al., 2010).

Phytase is a class of phosphatase that catalyzes sequential dephosphorylation of phytate by releasing phosphates, lower inositol phosphates and chelated components (Bohn et al., 2008; Kumar et al., 2010). Phytases can be found in plant and microbes as the 4/6-phytases (EC 3.1.3.26) and 3phytases (EC3.1.3.8), but it is absent in gastrointestinal tract of monogastric animals (Konietzny and Greiner, 2002; Bohn et al., 2008). Plant food offer good phytase source for monogastric animal in utilization of phytate complexes, as only minimal phytase activity is contributed by intestinal microflora (Pallauf and Rimbach, 1997). 
The phytate level in whole rice grain would depend on food matrix, co-occurrence of other components and agronomic conditions, which are generally categorized as genotype and environmental effects (Kussmann et al., 2007). The influence from genotype and environment are widely reported in cereal plants but their effects often varied (Liu et al., 2005; Polycarpe Kayodè et al., 2006; Dai et al., 2007; Mahmood et al., 2010). Other co-occurrence factors such as inherent phytase activity of whole grain rice could also influence the phytic acid level in whole grain rice. These highlighted the important of current study to clarify the influential factors of phytate content and phytase activity in local rice.

In this study, the variability of phytate content and phytase activity with genotypes, growing locations and grain morphology among thirty Bario rice cultivars from Sarawak, Malaysia was investigated. The determinant factor for the variability was also identified to provide a baseline reference for rice breeders and food processing industries in their nutritional improvement programs.

\section{Materials and Methods \\ Rice collection and samples preparation}

Thirty Bario rice cultivars were collected in paddy form from seven locations (Figure 1) in Limbang, Miri and Bintulu division, Sarawak, Malaysia. The Bario rice cultivars were referred to the rice cultivars named after Bario or Adan cropped in the mentioned growing locations. The paddy samples were dehusked manually using mortar and pestle to obtain whole grain rice. Pulverization was done with an electrical blender and sieved through a $425 \mu \mathrm{m}$ sieve. Moisture content was determined by using a moisture analyzer. Rice cultivars were grown and maintained for deoxyribose nucleic acid (DNA) extraction.

\section{Phytate content and phytase activity}

Phytate content determination was conducted as described by Dost and Tokul (2006). The determination of phytate content using high performance liquid chromatography (HPLC) was based on the complexometric replacement of ferric ion by phytic acid. The HPLC system was equipped with $\mathrm{CN} 3$ analytical column $(5 \mu \mathrm{m} ; 4 \times 150 \mathrm{~mm})$ manufactured by GL Science Inc. Chromatogram was monitored at $460 \mathrm{~nm}$ using a photodiode array detector. All cultivars were analyzed in triplicate with duplicate injections.

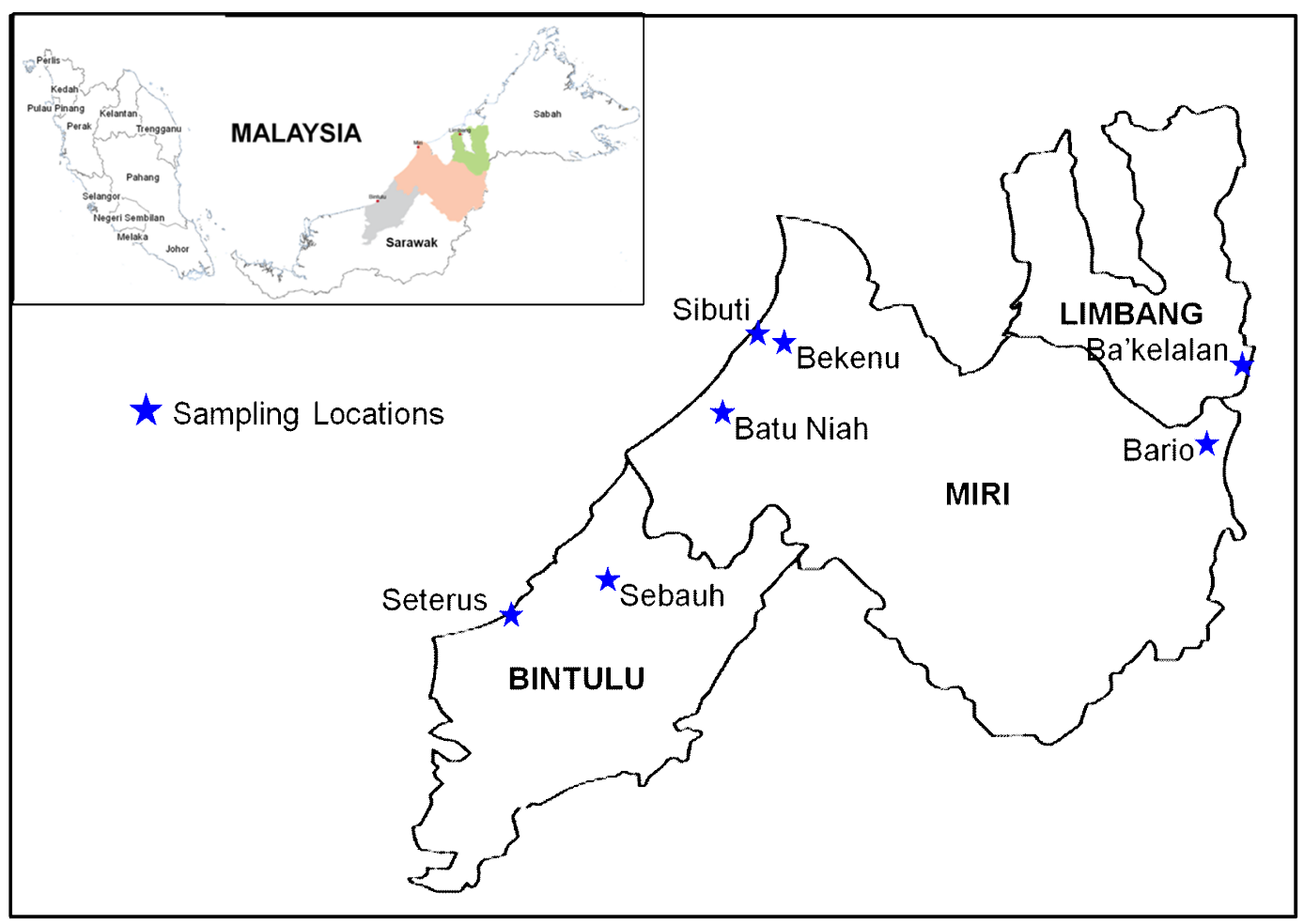

Figure 1. Sampling locations of the study. 
Phytase activity was determined as described by Kim and Lei (2005), based on the inorganic phosphorus released during incubation of phytase enzyme for a specified period of time. One unit phytase activity was defined as the amount of enzyme required to liberate one micromole of inorganic phosphate per minute from sodium phytate at $\mathrm{pH} 5.5$ and at $37^{\circ} \mathrm{C}$.

\section{Genetic profiles, grain morphology and growing locations}

Leaf samples were harvested from the 20 days old plants for DNA extraction and stored at $-80^{\circ} \mathrm{C}$. DNA was extracted according to the protocol of GF-1 Plant DNA Extraction Kit (Vivantis Technologies, USA). Polymorphism analysis was performed using the multiplex PCR technique, with four marker panels in triplex combination (Lee et al., 2010). The banding patterns were resolved in $10 \%$ polyacrylamide gel electrophoresis, running at 80 volts in a Bio-RAD Mini Protean system for 90 minutes.

Thousand kernels weight (TKW), grain length, grain width and lengths to width (L/W) ratios were characterized according to Juliano (1993). Grain size was deduced by measurement of grain length according Table 1. Grain shape was deduced from the ratio of grain length to width according to Table 2. Meteorological data was obtained from the meteorological department, drainage and irrigation department and soil survey department of Sarawak.

\section{Statistical analysis}

The statistical differences among cultivars and variation with genotypes, grain morphology and growing locations in phytate and phytase activity were estimated from ANOVA test, followed by Duncan New Multiple's Range Test (DMRT) using SAS Version 9.0 (2002; SAS Institute Inc., Cary,
NC, USA). Correlation analyses was tested by the Pearson's Student Correlation at the significant level of $\mathrm{P}=0.05$. The genetic relationship among the cultivars was inferred by a dendrogram generated using the NTSYSpc version 2.20r N software package (Rohlf, 2005).

Table 1. Grain length categories of whole grain rice.

\begin{tabular}{ll}
\hline Size & Length $(\mathrm{mm})$ \\
\hline Extra long & $>7.50$ \\
Long & $6.61-7.50$ \\
Medium & $5.51-6.60$ \\
Short & $<5.50$ \\
\hline
\end{tabular}

Table 2. Grain shape categories of whole grain rice.

\begin{tabular}{ll}
\hline Shape & Length/width ratio \\
\hline Slender & $>3.0$ \\
Medium & $2.1-3.0$ \\
Bold & $1.1-2.0$ \\
Round & $<1.0$ \\
\hline
\end{tabular}

\section{Results and Discussion Growing locations}

Farmers often share their paddy seeds among relatives or villages. Same cultivar may be cropped in different locations under various cropping practices and named according to the seed characteristics. In this study, a total of nine uplands and twenty one lowland Bario rice cultivars were collected from seven growing locations under 3 meteorological stations (Table 3). Ba'kalalan and Bario cropping location gave a large variation in surface air temperature as compared to lowland cropping location. The total rainfall amount and number of rain days between upland and lowland location was similar, except Batu Niah, Bekenu and Sibuti.

Table 3. Growing environmental conditions of sampling locations.

\begin{tabular}{llllll}
\hline $\begin{array}{l}\text { Meteorological } \\
\text { station }\end{array}$ & Sampling location & $\begin{array}{l}\text { Total rainfall } \\
\text { amount }(\mathrm{mm})\end{array}$ & $\begin{array}{l}\text { No. of rain } \\
\text { days }\end{array}$ & $\begin{array}{l}\text { Surface air } \\
\text { temperature }\left({ }^{\circ} \mathrm{C}\right)\end{array}$ & $\begin{array}{l}\text { Sunshine } \\
\text { hours }\end{array}$ \\
\hline Mulu & $\begin{array}{l}\text { Ba'kalalan } \\
\text { Bario }\end{array}$ & $143.7-473.4$ & $17-26$ & $22.6-34.8$ & n.a. \\
Miri & $\begin{array}{l}\text { Batu Niah } \\
\text { Bekenu }\end{array}$ & $7.8-453.6$ & $3-23$ & $26.1-27.6$ & n.a. \\
& $\begin{array}{l}\text { Sibuti } \\
\text { Sebauh }\end{array}$ & & & & \\
Bintulu & Seterus & $133.2-505.8$ & $10-26$ & $26.4-27.5$ & $5.0-7.3$ \\
& & & & & \\
\hline
\end{tabular}

n.a. indicates data not available. 
Table 4. Phytate content, phytase activity and grain morphology of 30 Bario rice cultivars from northern Sarawak, Malaysia.

\begin{tabular}{|c|c|c|c|c|c|c|c|c|}
\hline $\begin{array}{l}\text { Code } \\
\text { name }\end{array}$ & Cultivar name & Sampling site/Location & $\begin{array}{l}\text { Phytate content } \\
(\mathrm{mg} / \mathrm{g})\end{array}$ & $\begin{array}{c}\text { Phytase activity } \\
(\mathrm{U} / \mathrm{kg})\end{array}$ & $\begin{array}{l}\text { Max. length } \\
(\mathrm{mm})\end{array}$ & $\begin{array}{l}\text { Max. width } \\
(\mathrm{mm})\end{array}$ & $\begin{array}{l}\text { Length/width } \\
\text { ratio }\end{array}$ & $\begin{array}{c}\text { Thousand kernels } \\
\text { weight }(\mathrm{g})\end{array}$ \\
\hline SH01 & Adan Halus & Bario Asal/Bario & $22.67 \pm 2.10$ efghi & $57.13 \pm 1.01 \mathrm{cdefgh}$ & $5.96 \pm 0.28 \mathrm{cdef}$ & $1.71 \pm 0.09 \mathrm{a}$ & 3.5 & $12.63 \pm 0.64 q r$ \\
\hline $\mathrm{SH} 02$ & Adan Sederhana & Bario Asal/Bario & $20.92 \pm 2.13 \mathrm{ijk}$ & $28.55 \pm 1.99 \mathrm{jk}$ & $5.92 \pm 0.27 \mathrm{defg}$ & $1.93 \pm 0.08 \mathrm{def}$ & 3.1 & $13.56 \pm 0.03 p q$ \\
\hline $\mathrm{SH} 03$ & Adan Merah & Ulong Palang/Bario & $23.39 \pm 1.31$ efghi & $17.12 \pm 3.08 \mathrm{kl}$ & $6.47 \pm 0.22 \mathrm{cde}$ & $1.86 \pm 0.23 \mathrm{efg}$ & 3.5 & $16.74 \pm 0.38 \mathrm{op}$ \\
\hline $\mathrm{SH} 04$ & Adan Hitam & Bario Asal/Bario & $27.74 \pm 1.39 b c$ & $4.77 \pm 1.631$ & $6.10 \pm 0.42 b$ & $2.02 \pm 0.10 \mathrm{a}$ & 3.0 & $14.22 \pm 0.41 \mathrm{pq}$ \\
\hline SH05 & Adan Pulut & Bario Asal/Bario & $28.49 \pm 1.78 b$ & $51.97 \pm 5.99$ efghi & $6.89 \pm 0.30 \mathrm{a}$ & $2.18 \pm 0.11 \mathrm{cde}$ & 3.1 & $19.84 \pm 0.10$ no \\
\hline SH06 & Adan Tuan & Ulong Palang/Bario & $20.79 \pm 1.98 \mathrm{ijk}$ & $102.65 \pm 25.34 \mathrm{a}$ & $7.07 \pm 0.31 \mathrm{a}$ & $2.18 \pm 0.07 b c$ & 3.2 & $21.33 \pm 0.07 \mathrm{n}$ \\
\hline SH07 & Adan Halus & $\mathrm{Pa}^{\prime}$ Ukat/Bario & $26.67 \pm 3.80 \mathrm{bcd}$ & $57.89 \pm 1.89$ cdefgh & $6.05 \pm 0.28 \mathrm{cde}$ & $1.77 \pm 0.10 \mathrm{a}$ & 3.4 & $11.85 \pm 0.201$ \\
\hline SH08 & Adan & Ba'kalalan & $18.64 \pm 2.53 \mathrm{jk}$ & $71.33 \pm 1.73 b c$ & $5.63 \pm 0.26 \mathrm{hijk}$ & $1.97 \pm 0.10 \mathrm{ghijk}$ & 2.9 & $12.45 \pm 0.25 \mathrm{qr}$ \\
\hline SH09 & Adan Merah & Ba'kalalan & $22.33 \pm 2.17 \mathrm{ghi}$ & $54.85 \pm 10.21 \mathrm{defgh}$ & $5.90 \pm 0.21 \mathrm{efg}$ & $1.69 \pm 0.12 \mathrm{a}$ & 3.5 & $10.08 \pm 0.05 \mathrm{r}$ \\
\hline SL10 & Bario Pendek & Rh. Henry/Sebauh & $18.27 \pm 1.95 \mathrm{k}$ & $56.19 \pm 3.47$ cdefgh & $5.69 \pm 0.27$ ghijk & $2.10 \pm 0.14 \mathrm{klmn}$ & 2.7 & $14.44 \pm 0.05 \mathrm{pq}$ \\
\hline SL11 & Bario Banjal & Rh. Ruma/Seterus & $28.26 \pm 2.69 b c$ & $60.85 \pm 7.75 \mathrm{cdef}$ & $5.57 \pm 0.27 \mathrm{jk}$ & $2.09 \pm 0.10 \mathrm{mn}$ & 2.7 & $14.44 \pm 0.09 \mathrm{pq}$ \\
\hline SL12 & Adan Halus & Paya Selanyau/Bekenu & $21.62 \pm 4.07 \mathrm{hij}$ & $71.01 \pm 2.22 \mathrm{bc}$ & $5.77 \pm 0.21$ fghij & $1.73 \pm 0.07 b$ & 3.3 & $10.92 \pm 0.16 \mathrm{~m}$ \\
\hline SL13 & Adan Sederhana & Paya Selanyau/Bekenu & $25.67 \pm 1.74 \mathrm{bcdef}$ & $37.72 \pm 5.54 \mathrm{ij}$ & $5.49 \pm 0.23 \mathrm{k}$ & $2.10 \pm 0.09 \mathrm{mn}$ & 2.6 & $13.33 \pm 0.15 \mathrm{jk}$ \\
\hline SL14 & Bario & Kpg. Danau/Bekenu & $28.05 \pm 2.55 b c$ & $66.66 \pm 8.84 \mathrm{bcd}$ & $5.69 \pm 0.28$ ghijk & $2.11 \pm 0.07 \mathrm{lmn}$ & 2.7 & $14.30 \pm 0.33 \mathrm{hi}$ \\
\hline SL15 & $\operatorname{Bario}(\mathrm{A})$ & Rh. Unggeh/Sibuti & $18.20 \pm 2.64 \mathrm{k}$ & $48.73 \pm 9.00 \mathrm{fghi}$ & $5.93 \pm 0.40 \mathrm{defg}$ & $2.06 \pm 0.13 \mathrm{ghij}$ & 2.9 & $13.45 \pm 0.36 \mathrm{jk}$ \\
\hline SL16 & Bario (B) & Rh. Unggeh/Sibuti & $21.66 \pm 1.85 \mathrm{hij}$ & $59.10 \pm 18.83$ cdefg & $5.73 \pm 0.23$ fghijk & $1.98 \pm 0.09 \mathrm{ghi}$ & 2.9 & $13.22 \pm 0.30 \mathrm{k}$ \\
\hline SL17 & Bario Merah & Rh. Unggeh/Sibuti & $23.38 \pm 1.24$ efghi & $54.09 \pm 18.83$ defgh & $6.15 \pm 0.29 \mathrm{~cd}$ & $1.93 \pm 0.13 \mathrm{bcd}$ & 3.2 & $14.19 \pm 0.32 \mathrm{i}$ \\
\hline SL18 & Bario Sederhana & Kpg. Hunai/Bekenu & $22.61 \pm 3.06$ efghi & $55.87 \pm 5.25$ cdefgh & $5.85 \pm 0.41$ efghi & $2.22 \pm 0.07 \mathrm{mn}$ & 2.6 & $16.32 \pm 0.41 d$ \\
\hline SL19 & Bario Brunei & Rh. Ramang/Batu Niah & $22.39 \pm 2.35$ fghi & $79.04 \pm 1.83 \mathrm{~b}$ & $5.73 \pm 0.33$ fghijk & $2.09 \pm 0.09 \mathrm{ijklmn}$ & 2.7 & $13.66 \pm 0.03 \mathrm{j}$ \\
\hline SL20 & Bario Pendek & Niah/Batu Niah & $22.77 \pm 1.45$ efghi & $59.81 \pm 3.55 \mathrm{cdefg}$ & $5.87 \pm 0.23 \mathrm{efgh}$ & $2.06 \pm 0.12 \mathrm{hijkl}$ & 2.9 & $13.24 \pm 0.29 \mathrm{k}$ \\
\hline SL21 & Bario Pendek & Rh. Ramang/Batu Niah & $23.27 \pm 2.60$ efghi & $43.03 \pm 5.95 \mathrm{hij}$ & $5.75 \pm 0.27$ fghij & $2.20 \pm 0.09 \mathrm{mn}$ & 2.6 & $14.98 \pm 0.16 \mathrm{~g}$ \\
\hline SL22 & Bario Pendek & Rh. Tinggang/Batu Niah & $24.02 \pm 2.14 \mathrm{dfghi}$ & $65.52 \pm 4.51$ bcde & $5.77 \pm 0.45$ fghij & $2.11 \pm 0.12 \mathrm{jklmn}$ & 2.7 & $15.77 \pm 0.18 \mathrm{e}$ \\
\hline SL23 & Bario Brunei & Rh. Gamang/Batu Niah & $27.80 \pm 1.82 b c$ & $65.33 \pm 6.42$ bcde & $5.91 \pm 0.36 \mathrm{efg}$ & $2.25 \pm 0.13 \mathrm{mn}$ & 2.6 & $16.98 \pm 0.26 \mathrm{bc}$ \\
\hline SL24 & Bario Merah & Rh. Daud/ Batu Niah & $25.93 \pm 1.71 \mathrm{bcde}$ & $67.04 \pm 6.05 \mathrm{bcd}$ & $6.55 \pm 0.31 b$ & $2.21 \pm 0.12 \mathrm{fgh}$ & 3.0 & $16.67 \pm 0.24 c$ \\
\hline SL25 & Bario Merah & Rh. Gamang/ Batu Niah & $32.36 \pm 2.31 \mathrm{a}$ & $22.71 \pm 0.93 \mathrm{k}$ & $5.61 \pm 0.20 \mathrm{jk}$ & $2.14 \pm 0.08 \mathrm{mn}$ & 2.6 & $14.53 \pm 0.20 \mathrm{~h}$ \\
\hline SL26 & Bario Pendek & Rh Daud/ Batu Niah & $25.28 \pm 1.45$ bcdefg & $39.92 \pm 4.28 \mathrm{ij}$ & $5.56 \pm 0.19 \mathrm{jk}$ & $2.13 \pm 0.06 \mathrm{mn}$ & 2.6 & $14.13 \pm 0.08 \mathrm{i}$ \\
\hline SL27 & Bario Pendek & Rh. Gamang/ Batu Niah & $24.29 \pm 2.14 \mathrm{dfgh}$ & $56.23 \pm 0.95$ defgh & $6.54 \pm 0.74 b$ & $2.21 \pm 0.17$ fgh & 3.0 & $15.30 \pm 0.03 f$ \\
\hline SL28 & Bario Selepin & Rh. Gamang/ Batu Niah & $25.16 \pm 3.37$ cdefg & $40.64 \pm 1.80 \mathrm{ghi}$ & $5.93 \pm 0.38 \mathrm{defg}$ & $2.15 \pm 0.06 \mathrm{ijklm}$ & 2.8 & $14.07 \pm 0.17 \mathrm{i}$ \\
\hline SL29 & Bario Tinggi & Rh. Daud/ Batu Niah & $28.12 \pm 3.44 b c$ & $60.56 \pm 8.75 \mathrm{cdef}$ & $6.20 \pm 0.55 c$ & $2.41 \pm 0.13 n$ & 2.6 & $17.63 \pm 0.12 \mathrm{a}$ \\
\hline SL30 & Bario Tinggi & Rh. Usek/ Batu Niah & $25.44 \pm 3.80$ bcdefg & $93.91 \pm 8.66 \mathrm{a}$ & $5.87 \pm 0.33 \mathrm{efgh}$ & $2.41 \pm 0.10 \mathrm{o}$ & 2.4 & $17.25 \pm 0.16 b$ \\
\hline
\end{tabular}

Values reported were mean \pm standard deviation of five replications. Means in the same column with different letter (a-r) are statistically different (Duncan New Multiple's Range Test, $\mathrm{P}<0.05$. 
Emir. J. Food Agric. 2014. 26 (10): 844-852

http://www.ejfa.info/

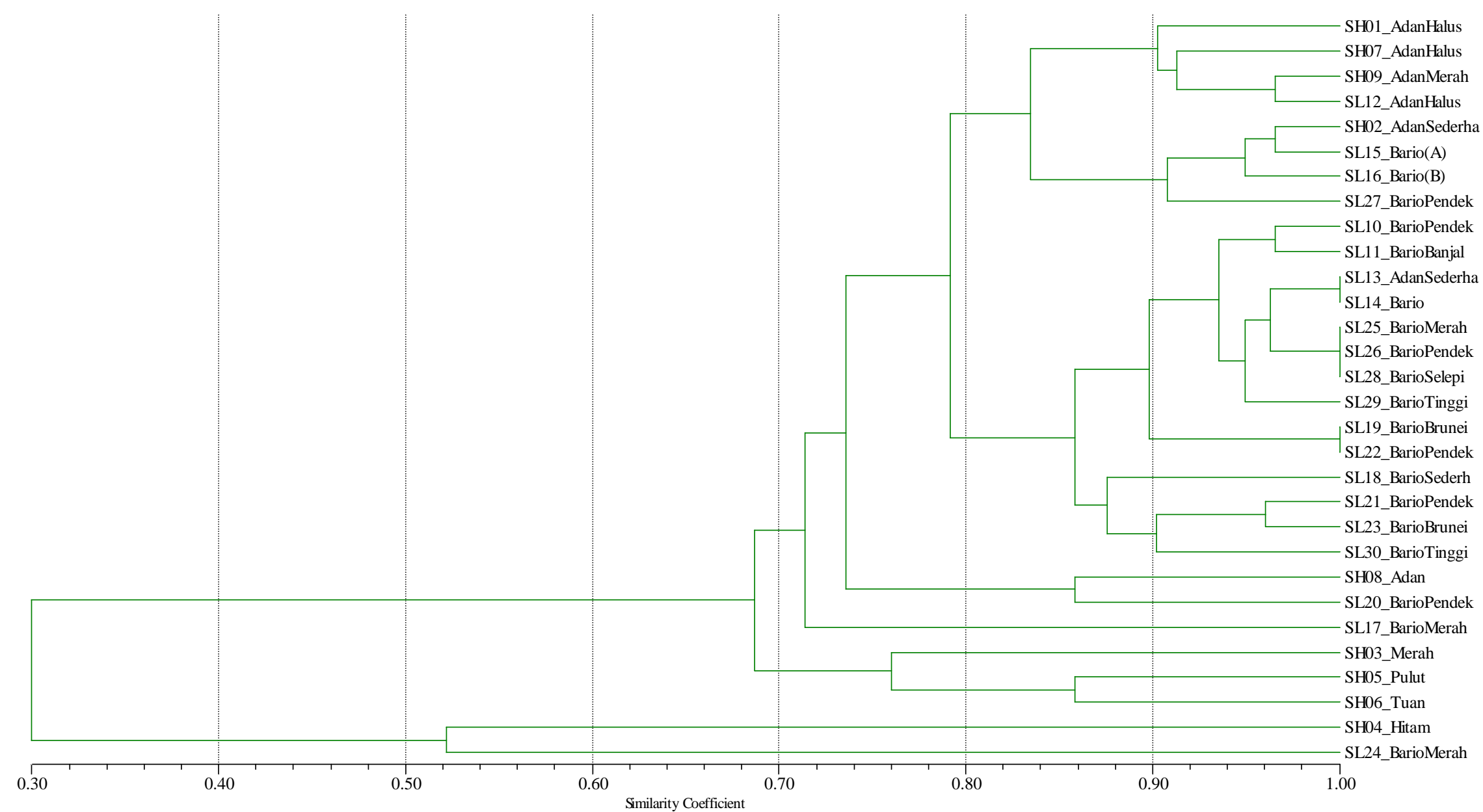

Figure 2. Genetic clustering of 30 Bario rice cultivars based on multiplex SSR marker panels (UPGMA, NTSYS-Pc). 
Emir. J. Food Agric. 2014. 26 (10): 844-852

doi: 10.9755/ejfa.v26i10.18503

http://www.ejfa.info/

\section{Grain morphology}

Grain morphology is the inherited characters of a variety (Wan et al., 2008; Bai et al., 2010). Different morphology (Table 4) was shown in the rice cultivars although they are given a similar name by farmers. The rice cultivars were differentiated into four physical grain morphology groups, i.e. medium-medium grain, mediumslender grain, long-slender grain and short-medium grain. Medium grain length and shape was the dominance group among the cultivars. Wide range of thousand kernels weights was shown with the values ranging from $10.08 \mathrm{~g}$ to $21.33 \mathrm{~g}$. Tuan and Pulut with genotypic similarity of $85.7 \%$ were found to be morphological distinct from the remaining rice cultivars.

\section{Genetic profiles}

Genotypic characterization allowed further differentiation of the rice cultivars, being claimed as Bario rice. The rice cultivars are differentiated into fourteen genotypes, consisting of five clusters and nine individual genotypes at $90 \%$ similarity based on UPGMA clustering (Figure 2). Genotype A consisting of four rice cultivars related to Adan Halus, which represents the popular rice in Bario Highlands. Adan Sederhana, the second major rice cultivars in Bario Highlands was clustered as genotype B. The largest cluster (genotype C) was consisting of lowland Bario rice cultivars, which includes the Adan Sederhana (SL13) adopted from Bario highlands. Genotypic difference was found between genotype $\mathrm{B}$ and $\mathrm{C}$, which could be due to the long term adaptation of Adan Sederhana in the lowland environment (Bajrasharya et al., 2006; Tu et al., 2007). Majority of the rice cultivars are closely related cultivars with similarity more than $70 \%$. The result showed that Adan Sederhana is the widely adopted highlands cultivar in lowland paddy field.

\section{Phytate content}

Phytate content and phytase activity are the parameters which could influence the antinutritional or antioxidant properties of the whole rice grain. Low phytate content and high phytase activity is often preferable due to wellknown anti-nutritional properties of phytic acid. Rice cultivars showed high phytate content (18.20 $32.36 \mathrm{~g} / \mathrm{kg}$, mean of $24.21 \mathrm{~g} / \mathrm{kg}$ ) with significant variation at $\mathrm{P} \leq 0.05$. The finding was comparable to Chinese rice $(7.2$ to $11.9 \mathrm{~g} / \mathrm{kg}$; mean of $9.6 \mathrm{~g} / \mathrm{kg}$ ), Chinese japonica rice (6.9 to $10.3 \mathrm{~g} / \mathrm{kg}$; mean of 8.7 $\mathrm{g} / \mathrm{kg}$ ) and Korean rice (8.6 to $17.6 \mathrm{~g} / \mathrm{kg}$; mean of
$12.6 \mathrm{~g} / \mathrm{kg}$ ) (Lee et al., 1997; Liu et al., 2005; Liang et al., 2007). High phytate content in rice cultivars implied some advantages in antioxidant properties, yet it showed high anti-nutritional effect in the whole grain.

Phytate content of the rice cultivars revealed a marginal significant variation $(\mathrm{P}<0.05)$ among growing locations (Table 5), with no significant correlation with grain morphology and genotype. Accordingly, phytate content was dependent on external factors of growing locations. However, meteorological conditions did not contribute to the influence of growing locations on phytate content. Different cropping and fertilization practices could be the influential factors due to diverse elevation and coastal distance among growing locations. Previous studies reported that phosphorus and zinc fertilization during the grain filling stage greatly accelerates the precipitation and accumulation of phytate (Raboy and Dickinson, 1984; Medeiros Coelho et al., 2002; Kaya et al., 2009).

\section{Phytase activity}

Phytase activity (4.77-102.65 U/kg, mean of $54.99 \mathrm{U} / \mathrm{kg}$ ) varied significantly among cultivars at $\mathrm{P}<0.05$. The phytase activity level was less than $200 \mathrm{U} / \mathrm{kg}$ and therefore categorised under low phytase cereal group (Eeckhout and De paepe, 1994). Different phytate content and phytase activity in rice could influence the degradation of the phytate during food ingestion. The low phytase activity could limit the phytate hydrolysis which in turns influences the anti-nutritional effect from phytic acid (Pallauf and Rimbach, 1997).

Phytase activity varied significantly with grain morphology and genotypes but not growing locations. Positive correlation between phytase activity, grain width and length to width ratio (Table 6) suggested that rounded grain had higher phytase activity. Thicker bran layer in short grain rice could facilitate the hydrolysis of phytate (del Rosario et al., 1968; Li and Ding, 2010). Highest phytase activity was represented by genotype L (Table 7). The influence of genotypes on phytase activity may be explained by the gene dependent synthesis of the phytase enzyme (Dionisio et al., 2011). Nevertheless, phytase activity did not significantly varied between the growing locations. These showed that phytase activity is largely controlled by intrinsic factors of genotypes and grain morphology. 
Table 5. Effect of growing locations on phytate content and phytase activity in Bario rice.

\begin{tabular}{llcll}
\hline Location & Cultivars & No. of cultivars & Phytate content $(\mathrm{mg} / \mathrm{g})$ & $\begin{array}{l}\text { Phytase activity } \\
(\mathrm{U} / \mathrm{kg})\end{array}$ \\
\hline Ba'kalalan & SH08, SH09 & 2 & $20.48 \mathrm{bc}$ & $63.09 \mathrm{a}$ \\
Bario & SH01 - SH07 & 7 & $24.38 \mathrm{abc}$ & $45.72 \mathrm{a}$ \\
Batu Niah & SL18-SL30 & 13 & $25.34 \mathrm{ab}$ & $57.61 \mathrm{a}$ \\
Bekenu & SL12-14 & 3 & $25.11 \mathrm{ab}$ & $58.46 \mathrm{a}$ \\
Sebauh & SL15-17 & 3 & $18.27 \mathrm{c}$ & $56.19 \mathrm{a}$ \\
Seterus & SL10 & 1 & $28.26 \mathrm{a}$ & $60.85 \mathrm{a}$ \\
Sibuti & SL11 & 1 & $21.08 \mathrm{bc}$ & $53.97 \mathrm{a}$ \\
\hline
\end{tabular}

Means in the same column with different letter (a-c) are statistically different (Duncan New Multiple's Range Test, $\mathrm{P}<0.05)$

Table 6. Phytate content and phytase activity of 14 genotype clusters from northern Sarawak Bario rice collection.

\begin{tabular}{clrrc}
\hline Genotype & Cultivars & $\begin{array}{l}\text { No. of } \\
\text { cultivars }\end{array}$ & $\begin{array}{l}\text { Phytate content } \\
(\mathrm{mg} / \mathrm{g})\end{array}$ & $\begin{array}{l}\text { Phytase activity } \\
(\mathrm{U} / \mathrm{kg})\end{array}$ \\
\hline A & SH01, SH07, SH09, SL12 & 4 & $23.32 \mathrm{a}$ & $60.22 \mathrm{abc}$ \\
B & SH02, SL15, SL16, SL27 & 4 & $21.27 \mathrm{a}$ & $48.15 \mathrm{bc}$ \\
C & SL10, SL11, SL13, SL14, SL25, SL26, & 8 & $26.39 \mathrm{a}$ & $48.16 \mathrm{bc}$ \\
& SL28, SL29 & & $25.51 \mathrm{a}$ & $67.22 \mathrm{ab}$ \\
D & SL21, SL23, SL30 & 3 & $23.21 \mathrm{a}$ & $72.28 \mathrm{ab}$ \\
E & SL19, SL22 & 2 & $22.61 \mathrm{a}$ & $55.87 \mathrm{bc}$ \\
F & SL18 & 1 & $18.64 \mathrm{a}$ & $71.33 \mathrm{ab}$ \\
G & SH08 & 1 & $22.77 \mathrm{a}$ & $59.81 \mathrm{abc}$ \\
H & SL20 & 1 & $23.38 \mathrm{a}$ & $54.09 \mathrm{bc}$ \\
I & SL17 & 1 & $23.39 \mathrm{a}$ & $17.12 \mathrm{~cd}$ \\
J & SH03 & 1 & $28.49 \mathrm{a}$ & $51.97 \mathrm{bc}$ \\
K & SH05 & 1 & $20.79 \mathrm{a}$ & $102.65 \mathrm{a}$ \\
L & SH06 & 1 & $27.74 \mathrm{a}$ & $4.77 \mathrm{~d}$ \\
M & SH04 & 1 & $25.93 \mathrm{a}$ & $67.04 \mathrm{ab}$ \\
\hline
\end{tabular}

Means in the same column with different letter (a-d) are statistically different (Duncan New Multiple's Range Test, $\mathrm{P}<0.05)$

Table 7. Pearson correlation matrix between phytate content, phytase activity and grain morphology.

\begin{tabular}{|c|c|c|c|c|c|c|}
\hline & $\begin{array}{l}\text { Phytate } \\
\text { content }\end{array}$ & $\begin{array}{l}\text { Phytase } \\
\text { activity }\end{array}$ & Length & Width & L/W Ratio & TKW \\
\hline Phytate content & 1 & -0.256 & 0.263 & 0.042 & 0.144 & 0.194 \\
\hline $\begin{array}{l}\text { Phytase } \\
\text { activity }\end{array}$ & & 1 & -0.164 & $0.509 *$ & $-0.538 *$ & 0.228 \\
\hline Length & & & 1 & 0.129 & $0.470 *$ & $0.666^{*}$ \\
\hline Width & & & & 1 & $-0.805^{*}$ & $0.699 *$ \\
\hline L/W Ratio & & & & & 1 & -0.247 \\
\hline TKW & & & & & & 1 \\
\hline
\end{tabular}

Values with asterisk (*) are significant at $\mathrm{P}<0.01$.

\section{Conclusion}

The whole rice grain was generally high in phytate content but low in phytase activity. The phytate content of the whole grain rice was locality dependent, while phytase activity was determined by their genotypes and grain morphology. Rice with rounded grain and in the genotype group of $\mathrm{L}$ (Tuan) showed a relatively high phytase activity. This enabled a dynamic phytase enzymatic activity rice could be selected for reducing their inherent phytate level, and enhancing their nutritional quality of whole grain.

\section{Acknowledgement}

We gratefully acknowledge the Ministry of Higher Education, Malaysia for their financial supports. Heartfelt thanks are also dedicated to the Department of Agriculture Sarawak, National 
Paddy Board, and Department of Irrigation and Drainage for their assistance and supports during the course of the study.

\section{References}

Bai, X., L. Luo, W. Yan, M. R. Kovi, W. Zhan and Y. Xiang. 2010. Genetic dissection of rice grain shape using a recombinant inbred line population derived from two constrasting parents and fine mapping a pleiotropic quantitative trait locus qGL7. BMC Genet. 11(1):11-16.

Bajracharya, J., K. A. Steele, D. I. Jarvis, B. R. Sthapit and J. R. Witcombe. 2006. Rice landrace diversity in Nepal: Variability of agro-morphological traits and SSR markers in landraces from a high altitude site. Field Crop Res. 95:327-335.

Bohn, L., A. S. Meyer and S. K. Rasmussen. 2008. Phytate: impact on environment and human nutrition. A challenge for molecular breeding. J. Zhejiang Univ-Sci. B 9(3):165-191.

Champagne, E. T., D. F. Wood, B. O. Juliano and D. B. Bechtel. 2004. The rice grain and its gross composition. In: E. T. Champagne (Ed.). pp. 77-100. Rice Chemistry and Technology 3rd edition. The American Association of Cereal Chemists.

Dai, F., J. Wang, S. Zhang, Z. Xu and G. Zhang. 2007. Genotypic and environmental variation in phytic acid content and its relation to protein content and malt quality in barley. Food Chem. 105:606-611.

del Rosario, A. R., V. P. Briones, A. J. Vidal and B. O. Juliano. 1968. Composition and endosperm structure of developing and mature rice kernel. Cereal Chem. 45:225.

Dionisio, G., C. K. Madsen, P. B. Holm, K. G. Welinder, M. Jørgensen, E. Stoger, E. Arcalis and H. Brinch-Pedersen. 2011. Cloning and characterization of purple acid phosphatase phytases from wheat, barley, maize and rice. Plant Physiol. 156(3):1087-100.

Dost, K. and O. Tokul. 2006. Determination of phytic acid in wheat and wheat products by reverse phase high performance liquid chromatography. Anal. Chim. Acta 558:2227.

Eeckhout, W. and M. De paepe. 1994. Total phosphorus, phytate-phosphorus and phytase activity in plant feedstuffs. Animal Feed Sci. Tech. 47(1):19-29.

Graf, E. and K. L. Empson. 1987. Phytic acid a natural antioxidant. Mol. Biol. 262(24):1164711650.

Jones, J. M. and J. Engleson. 2010. Whole grains: Benefits and challenges. Annu. Rev. Food Sci. Tech. 1:19-40.

Juliano, B. O. 1993. Rice in human nutrition. International Rice Research Institute, Philippines.

Kaya, M., Z. Küçükyumuk and I. Erdal. 2009. Phytase activity, phytic acid, zinc, phosphorus and protein contents in different chickpea genotypes in relation to nitrogen and zinc fertilization. Afr. J. Biotechnol. 8(18):45084513.

Kim, T. W. and X. G. Lei. 2005. An improved method for a rapid determination of phytase activity in animal feed. J. Animal Sci. 83:1062-1067.

Konietzny, U. and R. Greiner. 2002. Molecular and catalytic properties of phytate-degrading enzymes (phytases). Int. J. Food Sci. Tech. 37:791-812.

Kumar, V., K. A. Sinha, H. P. S. Makkar and K. Becker. 2010. Dietary roles of phytate and phytase in human nutrition: A review. Food Chem. 120:945-959.

Kussmann, M., M. Affolter, K. Nagy, B. Hols and L. B. Fay. 2007. Mass spectrometry in nutrition: understanding dietary health effects at the molecular level. Mass Spectrom. Rev. 26(6):727-50.

Lee, H. H., H. I. Rhee, S. Y. Lee, C. H. Kim and Y. S. Choi. 1997. Contents of phytic acid and minerals of rice cultivars from Korea. J. Food Sci. Nutr. 2:301-303.

Lee, H. H., S. C. Wong, A. Rajan, C. F. J. Bong and P. H. Yiu. 2010. Multiplex SSR Panels as Rapid Rice Genotyping Tool in Sarawak. Proceedings of the National Rice Conference 2010, Malaysia, pp. 390-393.

Li, Y. and W. Ding. 2010. Analysis on Microstructure Difference of Ventral and Dorsal Side of Rice Grain. Sci. Agric. Sinica 43(16):3473-3480.

Liang, J., B. Han, L. Han, M. J. Robert Nout and R. J. Hamer. 2007. Iron, zinc and phytic acid 
content of selected rice varieties from China. J. Sci. Food Agric. 87:504-510.

Liang, J., B. Han, M. J. Robert Nout and R. J. Hamer. 2008. Effects of soaking, germination and fermentation on phytic acid, total and in vitro soluble zinc in brown rice. Food Chem. 110:821-828.

Liu, Z., F. Cheng and G. Zhang. 2005. Grain phytic acid content in japonica rice as affected by cultivar and environment and its relation to protein content. Food Chem. 89:49-52.

Mahmood, T., T. Hameed, N. R. Siddiquiz, A. Mumtaz, N. Safdar and T. Masud. 2010. Effect of environmental changes on phytic acid content of wheat (Triticum aestivum). Pak. J. Nutr. 9(5):447-451.

Medeiros Coelho, C. M., J. C. Pires Santos, S. M. Tsai and V. Alexandre Vitorello. 2002. Seed phytate content and phosphorus uptake and distribution in dry bean genotypes. Braz. J. Plant Physiol. 14(1):51-58.

Norazalina, S., M. E. Norhaizana, I. Hairuszah and M. S. Norashareenaa. 2010. Anticarcinogenic efficacy of phytic acid extracted from rice bran on azoxymethane induced colon carcinogenesis in rats. Exp. Toxicol. Pathol. 62(3):259-268.

Oatway, L., T. Vasanthan and J. H. Helm. 2001, Phytic acid. Food Rev. Int. 17(4):419-431.

Pallauf, J. and G. Rimbach. 1997. Nutritional significance of phytic acid and phytase. Arch Tierernahr. 50(4):301-319.

Polycarpe Kayodea, A. P., R. Linnemann, R. H. Anita, D. Joseph, M. J. R. Nout and M. A. J. S. van Boekel. 2006. Genetic and environmental impact on iron, zinc and phytate in food - sorghum grown in Benin. J. Agric. Food Chem. 54:256-262.
Raboy V. and D. B. Dickinson. 1984. Effect of phosphorus and zinc nutrition on soybean seed phytic acid and zinc. Plant Physiol. 75(4):1094-1098.

Raboy, V., P. F. Gerbasi, K. A. Young, S. D. Stoneberg, S. G. Pickett, A. T. Bauman, P. P. N. Murthy, W. F. Sheridan and D. S. Ertl. 2000. Origin and seed phenotype of maize low phytic acid 1-1 and low phytic acid 2-1. Plant Physiol. 124:355-368.

Raboy, V., K. A. Young, J. A. Dorsch and A. Cook. 2001. Genetics and breeding of seed phosphorus and phytic acid. J. Plant Physiol. 158:489-497.

Rohlf, F. J. 2005. NTSYS-PC: Numerical taxonomy and multivariate analysis systems, Version 2.20r N. Exeter software, Setauket, New York.

Shahidi, F. 2009. Nutraceuticals and functional food: whole versus processed food. Trends Food Sci. Tech. 20(9):376-387.

Tu, M., B. R. Lu and Y. Zhu. 2007. Abundant within-varietal genetic diversity in rice germplasm from Yunnan Province of China revealed by SSR fingerprints. Biochem. Genet. 45:789-801.

Wan, X., J. Weng, H. Zhai, J. Wang, C. Lei, X. Liu, T. Guo, L. Jiang, N. Su and J. Wan. 2008. Quantitative trait loci (QTL) analysis for rice grain width and fine mapping of an identified QTL allele $\mathrm{gw}_{-5}$ in a recombination hotspot region on chromosome 5. Gen. Soc. America 179:2239-2252.

Yoon, J. H., L. U. Thompson and D. J. A. Jenkins. 1983. The effect of phytic acid on in vitro rate of starch digestibility and blood glucose response. Am. J. Clin. Nutr. 38:835-842. 